STAMPEDE-Studie

\title{
Op. bringt Diabetes in Remission
}

\author{
Bariatrische Chirurgie \\ verbessert bei adipösen \\ Diabetikern Gewicht und \\ Stoffwechsel nachhaltig.
}

Die Wirksamkeit der AdipositasChirurgie wurde in der Studie STAMPEDE belegt. Teilnehmer waren 150 adipöse Diabetiker (im Schnitt BMI 36). Zwei Drittel wurden operiert (Tunnelmagen oder Magenbypass), ein Drittel erhielt eine intensive antidiabetische Therapie mit regelmäßiger ärztlicher Beratung.

\section{Op. lässt Pfunde purzeln}

Binnen drei Jahren sanken in der Medikationsgruppe der HbAlc im Schnitt von 9\% auf $8,4 \%$ und das Gewicht um 4,3 kg. Nach
Magenbypass gingen der $\mathrm{HbA}_{1 \mathrm{c}}$ von 9,3\% auf $6,7 \%$ zurück und das Gewicht um $26 \mathrm{~kg}$, bei Tunnelmagen von 9,5\% auf 7\% $\left(\mathrm{HbA}_{\mathrm{lc}}\right)$ und $21 \mathrm{~kg}$ (Gewicht). Der Anteil der Patienten mit $\mathrm{HbA}_{\mathrm{lc}}$ unter 6\% lag nach drei Jahren bei $5 \%$ (medikamentöse Therapie), 38\% (Bypass) und 24\% (Tunnelmagen). Operierte Patienten mit $\mathrm{HbA}_{1 \mathrm{c}}$ unter $6 \%$ brauchten fast alle keine Antidiabetika mehr - sie waren ihren Diabetes los. (DE)

NEJM 2014; 370: 2002
- Reduktion des kardiovaskulären Risikos stehen (LDL-Ziel unter $100 \mathrm{mg} / \mathrm{dl}$, systolischer Blutdruck unter $140 \mathrm{mmHg}$, diastolischer: $80 \mathrm{mmHg}$ ).

- Wenn keine Kontraindikation vorliegt, ist Metformin das Medikament der ersten Wahl. Reicht dieses nicht (mehr) aus, ist die Kombination mit ein oder zwei oralen oder injizierbaren Medikamenten vernünftig, um die Nebenwirkungen gering zu halten.

- Schließlich wird bei vielen Patienten für eine angemessene Blutzuckerkontrolle Insulin nötig sein, entweder als Monotherapie oder in Kombination mit anderen Substanzen.

Besonders für die Wahl eines Antidiabetikums nach Metformin ist die Datenlage aber eingeschränkt, kritisiert Prof. Dr. Stumvoll.

EASD und ADA nennen hier nur die Vor- und Nachteile der geläufigen Substanzgruppen und überlassen den Ärzten die Auswahl.

- Für Sulfonylharnstoffe werden von den europäischen und den US-Diabetologen hohe Effektivität und niedrige Kosten vermerkt, allerdings auch ein mit- telschweres Risiko für Hypoglykämien sowie Gewichtszunahme.

- Die Effektivität der DPP-4-Hemmer wird als intermediär bezeichnet, das Hypoglykämierisko ist gering, weitere Nebenwirkungen sind selten. Die Behandlung damit ist gewichtsneutral; die Kosten sind hoch.

- Die GLP-1-Agonisten haben eine hohe Effektivität, das Risiko für Hypoglykämien ist gering und Patienten verlieren an Gewicht. Es gibt gastrointestinale Nebenwirkungen. Die Kosten der Therapie sind hoch.

- Insulin (in der Regel Basalinsulin) hat die höchste Wirksamkeit aller Antidiabetika. Das Hypoglykämierisiko ist hoch. Die Patienten nehmen zu. Die Kosten sind variabel.

Noch nicht berücksichtigt im Papier von EASD/ADA sind die SGLT-2-Hemmer. Sie werden aber etwa von der Diabetes-Gesellschaft in den Nationalen Versorgungsleitlinien sowohl in Monotherapie (bei Metformin-Unverträglichkeit) als auch zur Therapieeskalation empfohlen. (eis)

Diabetes Care 2012; 35: 1364

\section{ÜBRIGENS}

... leben Typ-1-Diabetiker mit Insulinpumpe länger als Diabetiker ohne. Das hat eine schwedische Analyse bei $2441 \mathrm{~Pa}$ tienten mit Pumpe und 15.727 mit normaler Insulintherapie ergeben, die beim EASD vorgestellt worden ist. Pumpenträger hatten im Vergleich ein um $29 \%$ geringeres Sterberisiko und ein um 44\% niedrigeres Risiko für tödliche kardiovaskuläre Ereignisse.

... ist eine Diabetes-Remission allein durch gesunden Lebensstil sehr selten. In einer Analyse bei 123.000 Typ-2-Diabetikern in den USA schafften in sieben Jahren 1,5\% eine Teilremission (HbA1c: 5,7-6,4\%) über zwölf Monate. Eine Vollremission $\mathrm{HbA} 1 \mathrm{c}<5,7 \%$ ) über fünf Jahre erreichten nur $0,001 \%$.

Diab Care 2014; 37; 3188

Punkte sammeln mit der Sommerakademie!

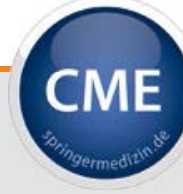

Zum Thema "Diabetes" finden Sie unter www.springermedizin.de/sommerakademie den Kurs:

Esstörungen bei Diabetes -

ein schlechtes Omen für die Prognose"

Mit e.Med teilnehmen

Mit e.Med haben Sie nicht nur Zugriff auf die aktuelle Sommerakademie, Sie können das komplette Angebot von Springer Medizin nutzen - zusätzlich erhalten Sie eine gedruckte Fachzeitschrift Ihrer Wahl. Mit rund 1600 Fortbildungskursen, davon über 500 CME-zertifiziert, ist die e.Akademie Deutschlands erste Adresse für Online-CME-Fortbildung.

Nutzen Sie die Sommerzeit, um reichlich CME-Punkte zu sammeln!

30 Tage kostenlos

Unser Kundenservice steht Ihnen wochentags (10-17 Uhr) kostenlos zur Verfügung: Tel. 08007780777 kundenservice@springermedizin.de 